\title{
High-throughput quantification of microbial birth and death dynamics using fluorescence microscopy
}

\author{
Samuel F. M. Hart, David Skelding ${ }^{\dagger}$, Adam J. Waite ${ }^{\dagger}$, Justin C. Burton ${ }^{\dagger}$, Wenying Shou* \\ Fred Hutchinson Cancer Research Center, Seattle, WA 98109, USA \\ * Correspondence: wenying.shou@gmail.com
}

Received June 16, 2018; Revised August 31, 2018; Accepted September 21, 2018

\begin{abstract}
Background: Microbes live in dynamic environments where nutrient concentrations fluctuate. Quantifying fitness in terms of birth rate and death rate in a wide range of environments is critical for understanding microbial evolution and ecology.

Methods: Here, using high-throughput time-lapse microscopy, we have quantified how Saccharomyces cerevisiae mutants incapable of synthesizing an essential metabolite (auxotrophs) grow or die in various concentrations of the required metabolite. We establish that cells normally expressing fluorescent proteins lose fluorescence upon death and that the total fluorescence in an imaging frame is proportional to the number of live cells even when cells form multiple layers. We validate our microscopy approach of measuring birth and death rates using flow cytometry, cell counting, and chemostat culturing.

Results: For lysine-requiring cells, very low concentrations of lysine are not detectably consumed and do not support cell birth, but delay the onset of death phase and reduce the death rate compared to no lysine. In contrast, in low hypoxanthine, hypoxanthine-requiring cells can produce new cells, yet also die faster than in the absence of hypoxanthine. For both strains, birth rates under various metabolite concentrations are better described by the sigmoidal-shaped Moser model than the well-known Monod model, while death rates can vary with metabolite concentration and time.

Conclusions: Our work reveals how time-lapse microscopy can be used to discover non-intuitive microbial birth and death dynamics and to quantify growth rates in many environments.
\end{abstract}

Keywords: Saccharomyces cerevisiae; fluorescence microscopy; microbial growth; birth rate; death rate

\begin{abstract}
Author summary: How fast microbes give birth or die (fitness) is influenced by their genetic makeups (genotypes) and environment. Microbes can mutate to many different genotypes, and the environment can change in many different ways. Thus, it is important to measure fitness for many genotypes in many environments so that we can understand, for example, why one genotype outcompetes another genotype. Here, we have developed a high-throughput method to quantify the fitness of fluorescent cells using time-lapse microscopy. We applied this method to two $S$. cerevisiae (budding yeast) mutants that had lost their ability to synthetize an essential metabolite. We found that the mutants behaved differently from one another in response to metabolite limitation, and in some cases, behaved differently from our expectations. Our method will be useful for quantifying growth phenotypes of fluorescent microbes.
\end{abstract}

${ }^{\dagger}$ These authors contributed equally to this work. 


\section{INTRODUCTION}

Microbial fitness are important to measure, since fitness differences between genotypes drive evolution and since fitness effects from other species are an important component of ecological interactions. Fitness needs to be measured in diverse conditions since microbes live in uncertain environments with, for example, fluctuating nutrient levels.

Fitness is often measured as the net growth rate (here referred to as "growth rate") - the difference between birth and death rates. An easy and rapid method for measuring growth rate is to track optical density of a culture over time. Since optical density cannot differentiate between live and dead cells, this method is useful when death rate is low. In contrast, flow cytometry can yield live and dead cell counts, but generally requires periodic manual sampling of the culture under observation. As an alternative method, high-throughput microscopy has been developed and applied to, for example, monitoring biofilm susceptibility to antibiotics [1] and quantifying growth rate heterogeneity among micro colonies [2]. High-content microscopy screening has been designed to track features such as cell shape and protein localization, and has been applied to libraries of deletion mutants or cells treated with RNAi or small molecule libraries $[3,4]$.

Here, we use microscopy to distinguish cell birth from cell death, especially at low metabolite concentrations where death rate is high. Distinguishing birth from death can be important. For example, metabolite consumption is tied to birth and not to death. As another example, in the extreme case of cells not dividing or dying, then natural selection ceases. Based on the same reasoning, if two populations have the same net growth rate, then a population that divides and dies slowly should evolve slower per unit time compared to a population that divides and dies rapidly.

Several mathematical models phenomenologically relate nutrient concentrations to population growth rates. The best known model is the Monod model [5], $g=g_{\max } s /\left(K_{M}+s\right)$, where $g$ is the growth rate, $s$ is the concentration of the limiting metabolite, $g_{\max }$ is the maximal growth rate, and $K_{M}$ is the concentration of $s$ at which half $g_{\max }$ is achieved. Other growth models such as the Teisser and the Contois [6] models have also been proposed. However, like the Monod model, they do not consider cell death since they assume zero (instead of negative) growth rate at zero metabolite concentration. A different growth model by Kovárová-Kovar and Egli [7] incorporates a fixed death rate, although in reality, death rate could vary with metabolite concentrations.

Previously, we constructed a two-strain synthetic yeast cooperative community as a model system to explore how cells in a cooperative community might evolve and how inter-species cooperation might shape species coexistence and spatial patterning [8-12]. In this community, a redfluorescent strain required lysine and released hypoxanthine (an adenine precursor) [13], while a greenfluorescent strain required hypoxanthine and released lysine. To establish a mathematical model for this community, we needed to measure each strain's birth and death rates at various concentrations of the required metabolite.

Here, we describe a microscopy assay that we developed and validated for quantifying a strain's birth and death rates at various concentrations of the required metabolite. Our approach can be applied to quantifying the birth and death dynamics of other fluorescently labeled microbes.

\section{RESULTS}

\section{Using fluorescence to quantify cell birth and death}

We imaged fluorescent yeast cells in microtiter plates. Our inverted fluorescence microscope was equipped with motorized stage and filter wheel, and was enclosed in a temperature-controlled chamber (Figure 1A; Methods, "Microscope setup") to ensure a nearly constant temperature (Supplementary Figures S1 and S2). To enable automated long-term imaging with minimal photodamage, we wrote a LabVIEW routine to perform autofocusing in bright-field (Methods, "Autofocusing") and then capture images in the fluorescence channel. However, despite controlling the chamber temperature, condensation developed on the microplate lid over time, which sometimes interfered with autofocusing. To resolve this, we developed a "lid warmer" (Methods, "Lid warmer") using transparent, conductive ITO glass (Figure 1B) to warm the microplate lid to $\sim 0.7^{\circ} \mathrm{C}$ above the stage temperature (Supplementary Figure S1B). This eliminated condensation (Figure 1C) and allowed reliable auto-focusing over tens of hours.

For yeast cells expressing a fluorescent protein, total fluorescence intensity (after background subtraction) scaled linearly with live fluorescent cell density up to at least nine cell layers (Supplementary Figure S3). Cells lost fluorescence immediately upon losing cell integrity (compare Supplementary Movie 3 vs Supplementary Movie 4). Thus, increases or decreases in fluorescence were proportional to cell division or cell death, respectively.

To measure birth and death rates, we performed timelapse imaging of fluorescent yeast cells at various concentrations of the required metabolite (Figure 1D). Even in the absence of the required metabolite, total fluorescence intensity initially increased due to residual 
A

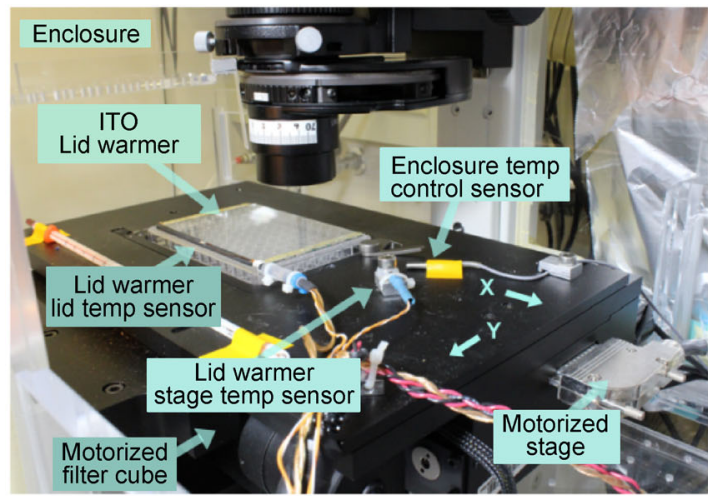

D

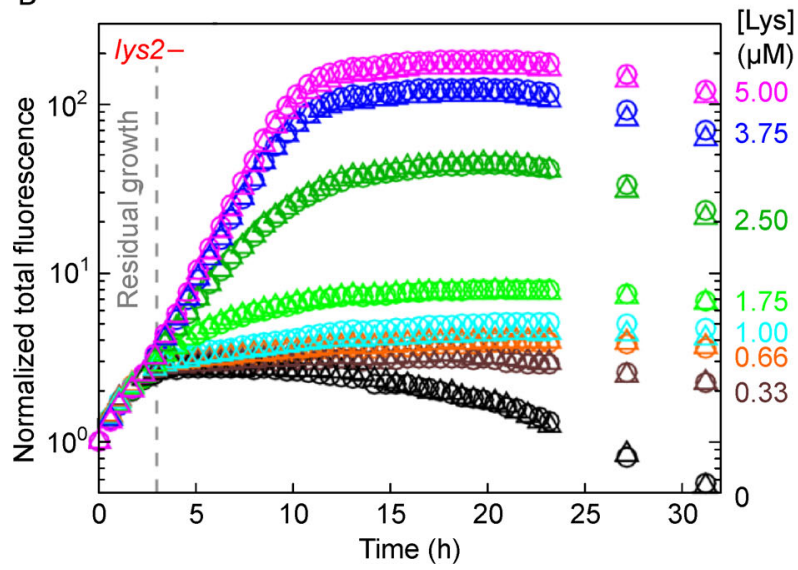

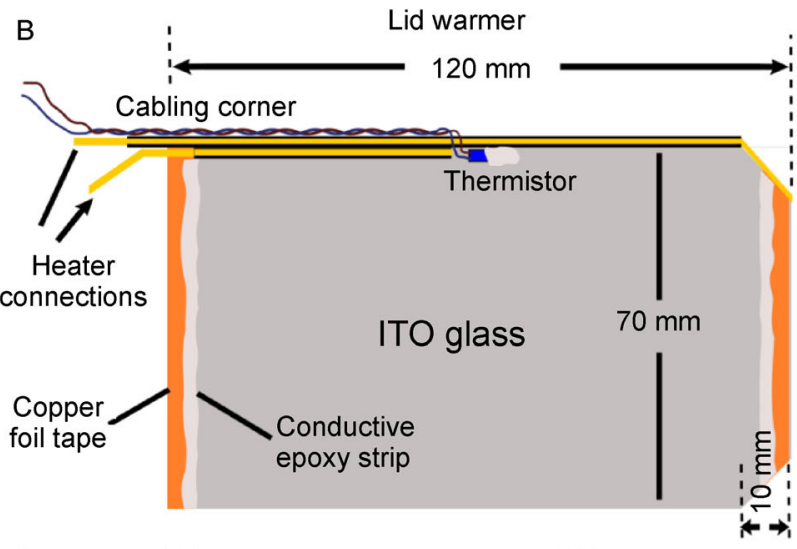

C

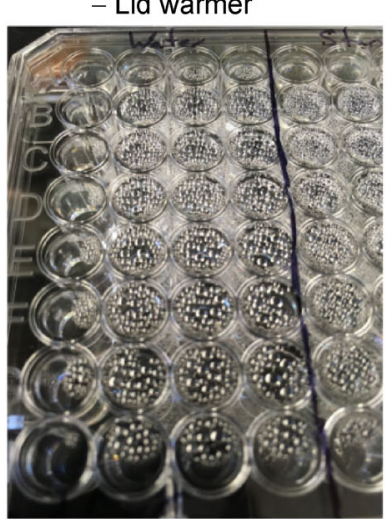

+ Lid warmer

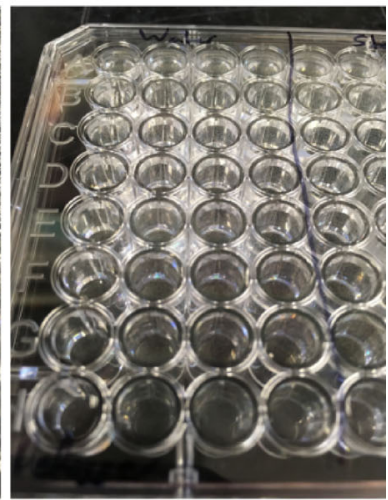

Figure 1. Automated high-throughput microscopy. (A) Microscope setup. An enclosure around the microscope provides a temperature-controlled environment. A motorized stage allows repeated bright-field and fluorescent imaging of the same positions in specified wells of a microtiter plate (Supplementary Movies 1-3). Motorized filter cubes allow automated filter switching. (B) An ITO glass lid warmer prevents condensation. Sensors on the plate lid ("thermistor") and the microscope stage provide temperature measurements to a LabVIEW program which turns the ITO lid warmer on or off to maintain the plate at $\sim 0.7^{\circ} \mathrm{C}$ warmer than the stage (Supplementary Figure S1). (C) The lid warmer eliminates condensation. The images were taken after a 24-hour imaging experiment at $30^{\circ} \mathrm{C}$. (D) Growth of lys2- (WY1335) cells at various lysine concentrations. Background-subtracted total fluorescence intensities from four picture frames were normalized against their respective initial values, averaged, and plotted. Residual growth (prior to dashed line) at zero lysine is presumably fueled by vacuolar lysine storage. When calculating growth rates, we only considered post residual growth data. Circles and triangles mark two independent experiments.

growth fueled by cellular storage of metabolites [14] (e.g., $0-3 \mathrm{~h}$ in Figure 1D). Thus for our data analysis, we only used images after residual growth.

\section{Death rate is time-dependent}

Death rate is not constant. We measured death rate of lys 2 - cells in zero lysine. Since new birth was negligible (no birth out of 603 cells over 30 h; e.g., Supplementary Movie 3) and since total fluorescence was indicative of live cell density, death rate could be estimated by quantifying the negative slope of $\ln$ (fluorescence) against time. We observed multi-phasic death kinetics, with a slow death rate followed by a faster death rate (Figure 2A, lightest grey; Figure 2B, [Lys]=0; Supplementary
Figure S4). ade8- cells in the absence of hypoxanthine also displayed time-dependent death rate (Figure 2C and 2D). For both strains, death rate would eventually slow down, as demonstrated in our previous work [8]. As shown below, death rate also depended on metabolite concentration.

Death rates from independent quantification methods are largely comparable. We quantified death rates of lys2cells in zero lysine by flow cytometry, and by manual or automated counting of live fluorescent cells in microscopy images, and compared results to that from quantifying total fluorescence intensity (Figure 2B, [Lys] $=0$; Supplementary Figure S4; Methods, "Individual cell tracking at low metabolite concentrations"). As expected, subtle differences existed among the three 

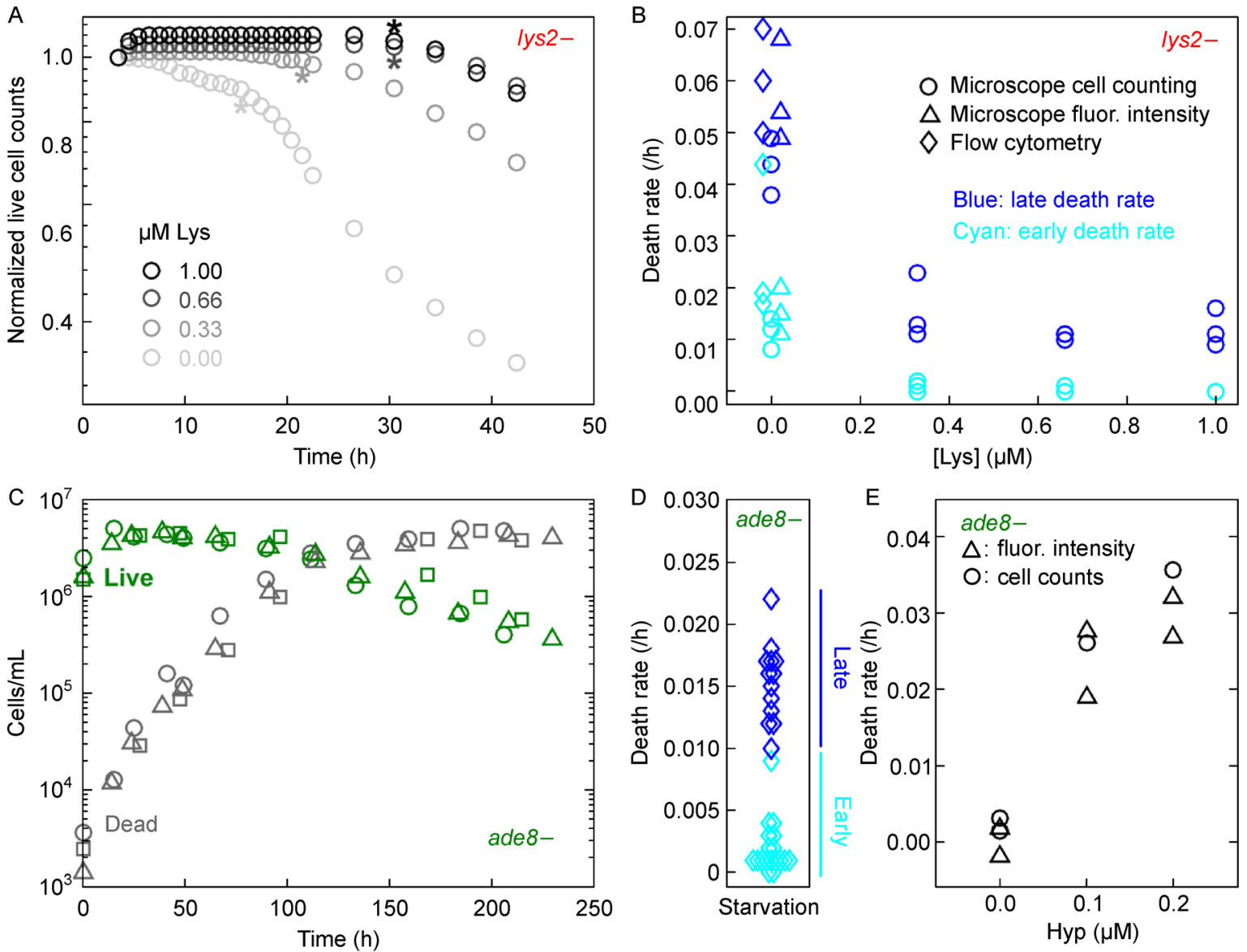

Figure 2. Death rate is nutrient- and time-dependent. Exponentially-growing lys2- (WY1335) cells were washed free of lysine at $0 \mathrm{~h}$, and imaged in minimal medium SD supplemented with 0 to $1 \mu \mathrm{M}$ lysine. (A) Birth events were restricted to the first few hours, and thus may be regarded as part of residual growth. Early and late death rates were calculated using data before and after asterix $\left.{ }^{*}\right)$, respectively, and plotted in panel B (circles). (B) Death rates of lys2-cells at $0 \mu \mathrm{M}$ lysine were also tracked using microscopy fluorescence intensity (triangles) and flow cytometry (diamonds). In all assays, death rate started slow (cyan), and then increased (blue). Death rates from the three quantification methods were comparable, although manual cell counting seemed to report lower death rate than the other two methods (circles lower than triangles and diamonds of the same color). For each experiment, we tracked birth and death events from an initial of 200-300 cells. Death rates at $0 \mu \mathrm{M}$ lysine were jittered along the $x$-axis to aid visualization. (C) Death dynamics of ade8- cells. Exponential ade8- (WY1340) cells were washed free of hypoxanthine supplements at time zero. Because ade8- cells died slowly, evaporation from small sample volume in microscopy assay became significant. Thus, we incubated a larger culture volume in a glass tube and tracked dead and live cell densities over time using flow cytometry. (D) Multiphasic death rate. Death rate was initially slow (cyan) and then sped up (blue). Early death rates were generally calculated within 24-96 h, while late death rates were generally calculated within 70-230 h. (E) Death rates of ade8-cells in zero or low concentrations of hypoxanthine. Exponential ade8- (WY1340) cells were washed free of hypoxanthine supplements at time zero. Death rate was quantified from $24 \mathrm{~h}$ to $42-66 \mathrm{~h}$ either by counting live cells or measuring total fluorescence intensity over time. In cases where we observed mutant clones, we excluded those from our quantification.

methods. Overall, manual cell counting yielded a slightly higher death rate than automated cell counting (Supplementary Figure S5B), presumably because manual but not automated cell counting could detect the death of a bud. Manual cell counting yielded a lower death rate than flow cytometry (Figure 2B, compare circles with diamonds of the same color), possibly because sample handling in flow cytometry reduced the viability of starving cells. Finally, manual cell counting yielded a slightly lower death rate than fluorescence intensity (Figure 2B, compare circles with triangles of the same color). This implies that fluorescence per live cell declined during starvation.

\section{Distinct birth and death dynamics at low metabolite concentrations}

Low concentrations of metabolites may not be consumed. We started with a small number of cells to minimize 
metabolite depletion during growth rate measurements in low concentrations of metabolite (Methods, "Sample setup"). Low concentrations of lysine (e.g., 0.33-1 $\mu \mathrm{M}$; Supplementary Movie 2) were barely consumed by lys $2-$ cells, while concentrations $>1.75 \mu \mathrm{M}$ were depleted down to $<1 \mu \mathrm{M}$ (Figure 3B). Consistently, maximal fluorescence intensity only scaled linearly with input lysine concentrations greater than $1.75 \mu \mathrm{M}$ (Figure 3A), the level around which half maximal growth rate was achieved (Figure 4). We inferred that low concentrations of hypoxanthine (e.g., $0.1-0.2 \mu \mathrm{M}$; too low to be directly measured from supernatants) were largely un-consumed by ade 8 - cells based on the following inference. Since 1-3 fmole of hypoxanthine was consumed per ade8 - cell [13], the input medium $\left(300 \mu \mathrm{L}\right.$ of $0.2 \mu \mathrm{M}$ or $6 \times 10^{4}$ fmole hypoxanthine) should support the birth of a total of $2 \times 10^{4}-6 \times 10^{4}$ cells. Instead, we observed an increase of $\leqslant 1800$ cells $(\leqslant 60 \%$ of the starting 3000 cells; Figure 3C).

Low concentrations of metabolites lead to distinct birth and death dynamics depending on the strain genotype. For lys 2 - cells in low $(\leqslant 1 \mu \mathrm{M})$ lysine, although total fluorescence intensity increased for longer compared to zero lysine (Figure 1D), this increase generally corresponded to cell swelling rather than cell birth (Supplementary Figure S7). Birth events, if any, were restricted to the initial few hours and not sustained at later time points (Figure 2A and Supplementary Figure S6A) despite the nearly-constant metabolite concentration (Figure 3B). Thus, the initial birth events of lys 2 - could be interpreted as low lysine prolonging the residual growth phase and delaying the onset of death phase. Low lysine also reduced early and late death rates of lys 2 - cells (Figure 2B). In contrast, for ade8- cells, low input concentrations (e.g., $0.1-0.2 \mu \mathrm{M}$ ) of hypoxanthine led to increased birth and death rates compared to zero hypoxanthine (Figure 3C). ade8- cells were occasionally born in low hypoxanthine, even after the onset of death phase (Supplementary Figure S6B from 30 to $66 \mathrm{~h}$ ). Moreover, a small number of ade 8 - cells transiently lost fluorescence, but then regained fluorescence and continued to divide (Supplementary Movie 5). In summary, for lys2- cells, low concentrations of lysine were not consumed, did not sustain birth beyond the initial stage, but delayed the onset of death phase and slowed the death rate once cells began to die (Figure 2). For ade8- cells, low concentrations of hypoxanthine, which were likely largely unconsumed, increased both birth and death rates (Figure 3C). Overall, death rate is not only timedependent, but also nutrient concentration-dependent.

\section{Microscopy and chemostats yield similar results at intermediate metabolite concentrations}

A concern about microscopy assay is that growth rate at a particular input metabolite concentration will continuously decline as the metabolite is consumed. To test this, we grew lys 2 - cells in various input lysine concentrations, and measured growth rate (e.g., slope in Figure 1D) across sliding time windows (Supplementary Figure S8). For lysine concentrations $\geqslant 2.5 \mu \mathrm{M}$, maximal growth rate was maintained across at least two contiguous sliding windows (Supplementary Figure S8), and thus can be used as the growth rate corresponding to the input lysine concentration. However, at lower lysine concentrations (e.g., $1.75 \mu \mathrm{M}$ ), the growth rate continuously declined throughout the experiment, and thus it was unclear whether our measured maximal growth rate was truly maximal. To verify our observations, we employed an independent measurement method where we grew lys2-
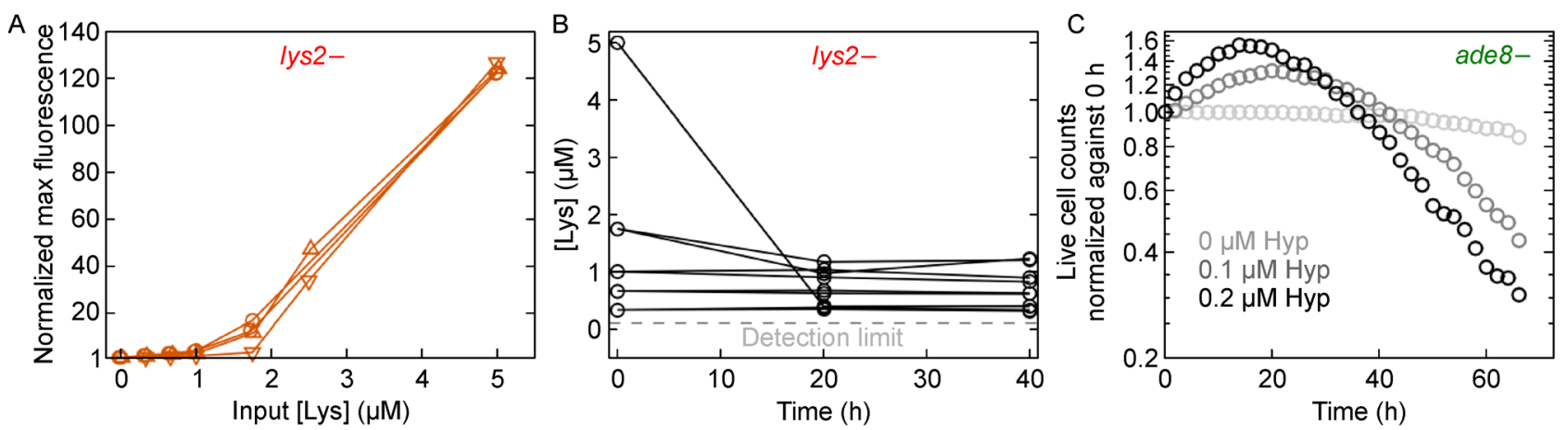

Figure 3. Very low concentrations of metabolites may not be consumed and can lead to distinct birth and death dynamics. (A) Maximal fluorescence of a lys2 - (WY1335) culture scales with input lysine concentration only when lysine concentration exceeds a threshold $(1.75 \mu \mathrm{M})$. Maximal fluorescence was normalized against that at zero lysine. (B) Low concentrations of lysine $(\leqslant 1 \mu \mathrm{M})$ remain largely unconsumed by lys 2 - over $40 \mathrm{~h}$. We measured lysine concentrations in supernatants using the rate bioassay (Methods, "Bioassay"). (C) A low level of hypoxanthine increases cell birth and death in ade8- cells. Exponential ade8- (WY1340) cells were washed and starved for $24 \mathrm{~h}$, and then imaged every $2 \mathrm{~h}$ in $0 \mu \mathrm{M}$ (light grey), $0.1 \mu \mathrm{M}$ (dark grey) and $0.2 \mu \mathrm{M}$ (black) hypoxanthine. A small amount of hypoxanthine increased both cell birth (0-20 h; Supplementary Figure S6B) and cell death (20-66 h). 
cells in lysine-limited chemostats [15] where population growth rate could be controlled by setting the dilution rate (Methods, "Chemostat culturing"). We set the dilution rate to various values, and measured the corresponding lysine concentrations (Supplementary Figure S9). Chemostat measurements were consistent with microscopy measurements (Figure 4A, blue crosses).

\section{The Moser model is superior to the Monod model in describing lys 2 - and ade - - cell birth}

The birth rate of lys 2 - cell increased with lysine concentration in a sigmoidal fashion. At low $(\leqslant 1 \mu \mathrm{M})$ lysine, birth rate was zero (Figure 2A; Supplementary Figure S6A). When lysine was not rate-limiting $(\geqslant 5$ $\mu \mathrm{M})$, lys 2 - cells achieved a maximal growth rate of $0.50 \pm 0.02 / \mathrm{h}$ in microscopy assay (Figure 1D, magenta), consistent with the value measured from culture optical density over time $(0.49 \pm 0.03 / \mathrm{h})$. At intermediate and high lysine concentrations $(\geqslant 1.75 \mu \mathrm{M})$, death rate was small $(<0.002 / \mathrm{h}[13])$ compared to growth rate $(\geqslant 0.1 / \mathrm{h})$, and thus we approximated growth rate as birth rate (Figure 4A). The data were better characterized by the Moser model $b(s)=b_{\max } s^{n} /\left(K_{m}{ }^{n}+s^{n}\right)$ (black line; Figure 4A), than the Monod model $b(s)=b_{\max } s /\left(K_{m}+\right.$ $s$ ) (grey dotted line; Figure 4A). In both models, $s$ is the lysine concentration, $b_{\max }$ is the maximal birth rate, $K_{m}$ is the Monod constant ( $s$ required to achieve half maximal birth rate). The Moser model has an additional parameter, $n$, which is analogous to the cooperativity coefficient in the Hill equation. For ade8-, the Monod model worked relatively well, but the Moser model provided a more accurate estimation of the maximal birth rate (Supplementary Figure S10 legend). Increased accuracy in the Moser model is not surprising given the additional parameter. Nevertheless, an accurate fit to experimental data is useful when we incorporate the relationship between metabolite concentrations and growth rates into population dynamics models.

\section{DISCUSSION}

Using microscopy, we quantitatively measured cell growth in a wide range of nutrient environments. Growth rates can differ depending on the duration of starvation (Supplementary Figure S11), where we cut off "residual growth" (Figure 2A and Supplementary Figure S8), and whether birth and death rates are calculated from fluorescence or cell counts (Figure 2B and Figure 3C). Since there is no single "correct" procedure, it is

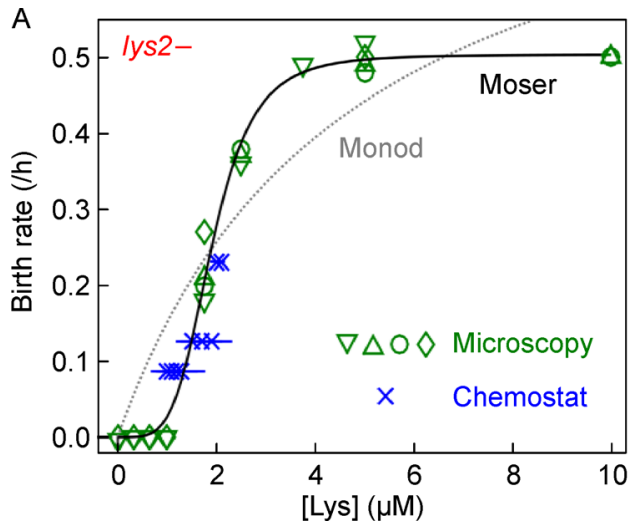

B

High lysine $(164 \mu \mathrm{M}) \quad$ Low lysine $(1 \mu \mathrm{M})$

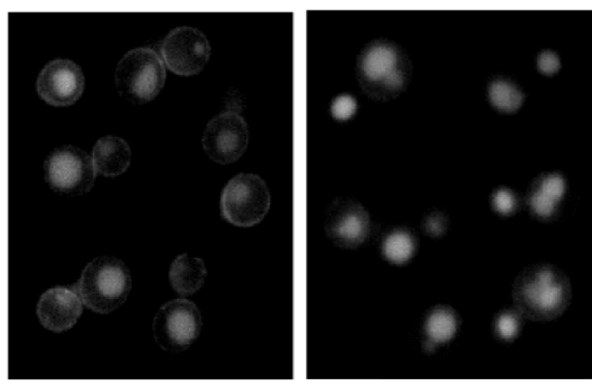

Figure 4. The birth rate of lys2- as a function of lysine concentration is better described by the Moser model than the Monod model. (A) A sigmoidal relationship between birth rate and lysine concentration. For lysine concentrations $\leqslant 1 \mu \mathrm{M}$, birth rates were zero (Supplementary Figure $S 6 \mathrm{~A}$ ). For lysine concentrations $\geqslant 1.75 \mu \mathrm{M}$, we calculated growth rates within $2-4 \mathrm{~h}$ sliding windows and used the maximal growth rates to approximate birth rates since death rates were small ( 0.002/h [13]). Separately in chemostats, we controlled growth rates ( birth rates) and measured the corresponding lysine concentrations at steady state (Methods). Results from microscopy (green) and chemostats (blue) are consistent. The birth rate of lys2- as a function of lysine concentration can be described by the Moser equation (black) where $b(L)=b_{\max L} L^{n_{L}} /\left(K_{L}^{n_{L}}+L^{n_{L}}\right)$ with $b_{\max L}=0.50(95 \% \mathrm{Cl}$ : 0.48 $0.53)$ per hour, $K_{L}=1.9(95 \% \mathrm{Cl}: 1.8-2.0) \mu \mathrm{M}$, and $n=4.47$ (95\% Cl: 3.2-5.7). In comparison, the best-fit of the Monod equation $g(L)=b_{\max L} L /\left(K_{L}+L\right)$ (grey dotted, $b_{\max L}=0.85$ per hour and $K_{L}=4.6 \mu \mathrm{M}$ ) fits the data poorly. (B) Lysine permease Lyp1 is localized to the vacuole in response to low lysine. We tagged the high-affinity lysine permease Lyp1 with a fluorescent GFP tag in lys2-cells (WY1620) to observe Lyp1 localization. Cells were grown to exponential phase in SD plus $164 \mu \mathrm{M}$ lysine, and washed and resuspended in SD plus $1 \mu \mathrm{M}$ or $164 \mu \mathrm{M}$ lysine. Cells were diluted into wells of a Nunc 96-well Optical Bottom Plate (Fisher Scientific, 165305) and imaged after $10 \mathrm{~h}$ using a $40 \times$ oil immersion objective, but otherwise under standard microscopy conditions (Methods). Lyp1-GFP localized to the membrane and the vacuole in high lysine (left), but localized predominately to the vacuole in low lysine (right). 
important to be aware of the limitations of each method. We recommend cross-checking one method against another, independent, method. For example, we crosschecked microscopy measurements against chemostat measurements (Figure 4), and cross-checked microscopy measurements against flow cytometry (Figure 2B and Supplementary Figure S4).

Population dynamics of lys 2 - and ade - strains shared certain features under nutrient limitation. For example, both displayed time- and nutrient-dependent death rates (Figure 2 and Supplementary Figure S4). The two strains differed in other aspects. For lys $2-$ cells, low concentrations $(0.33-1 \mu \mathrm{M})$ of lysine were largely un-consumed (Figure 3A and 3B), and did not support birth beyond residual growth (Supplementary Figure S6A), but did delay and slow death when compared to full starvation (Figure 2). In contrast, for ade8 - cells, low concentrations $(0.1-0.2 \mu \mathrm{M})$ of hypoxanthine increased death rates (Figure 3C) compared to full starvation, and, at the same time, supported some birth (Supplementary Figure S6B). These seemingly counter-intuitive behaviors probably resulted from the fact that these mutations disrupted biosynthetic pathways that would normally produce the necessary metabolite. In the case of ade8-, the diverse forms of adenine-containing compounds in the cell (including those in RNA and DNA, ATP, ADP, AMP, and reduced and oxidized forms of NAD, NADP, and FAD) possibly allowed new births to occur. In the case of lys 2-, the storage might be more limited and new births did not occur beyond residual growth. In both cases, since the cell did not "know" that it could not make the metabolite, it would try to grow and divide in the absence of the metabolite, which resulted in an abnormally high death rate [16].

The Monod model has been observed to fit, for example, the growth rates of yeast strains at various glucose concentrations [2]. For lys $2-$ cells, since the birth rate of lys2- increased with lysine concentration in a sigmoidal fashion, the Moser model fit the data much better than the Monod model (Figure 4A). This sigmoidal relationship could be explained by, for example, cooperative binding by nutrient transporters, as seen in a variety of cells types, including yeast [17-19]. However, in the case of lys 2 - cells, this sigmoidal relationship likely resulted from stress-induced permease re-cycling. Specifically, at very low lysine concentrations, high affinity lysine permease Lyp1 was ubiquitinated and targeted to the vacuole for degradation [20] (Figure 4B) so that the amino acids could be recycled to help cells cope with the stress [21].

For ade8- cells in hypoxanthine, the Monod model closely fit experimental data. The Moser model was still more accurate than Monod model (Supplementary Figure $\mathrm{S} 10$ ), although a two-parameter model (Moser) is expected to improve the fit over a one-parameter model (Monod). Regardless, a growth model that faithfully captures experimental observations is useful. For example, when modeling a community of two cross-feeding strains, a Moser model of how fast each strain grows in various concentrations of partner-supplied metabolite can be incorporated into the community dynamics model.

In summary, our work demonstrates the potential of high-throughput microscopy assays in quantifying microbial birth and death dynamics. Our method can be used to, for example, estimate the chronological life span of individual cells in a high-throughput fashion.

\section{METHODS}

\section{Strains and growth medium}

We used strains from the RM11 background with the following genetic modifications introduced via transformation [22]. Strain WY1335 ("lys2-") has the genotype of ho::loxP AMN1-BY ste3::Hph fba1::FBA1-mCherryloxP ade $4:: A D E 4-P U R 6$ (ole) lys $2::$ loxP. Strain WY1340 ("ade8-)" has the genotype of ho::loxP AMN1-BY ste3:: NAT fba1::FBA1-EGFP-loxP lys21::LYS21(o/e) ade8:: $\operatorname{lox} P$. For our bioassay of low metabolite concentrations, we used an evolved clone (WY2270) isolated after lys2had grown for tens of generations under lysine limitation. This clone displayed an increased affinity for lysine. For our LYP1 localization assay, we tagged LYP1 with GFP in a lys2 - strain via transformation (WY1620). We stored these strains at $-80^{\circ} \mathrm{C}$ in YPD $+15 \%$ glycerol.

We used rich medium YPD $(10 \mathrm{~g} / \mathrm{L}$ yeast extract, $20 \mathrm{~g} / \mathrm{L}$ peptone, $20 \mathrm{~g} / \mathrm{L}$ glucose) for streaking out single colonies and for growing saturated YPD overnight cultures which were then used as inoculum to grow exponential cultures. We found ade8- cells could grow to a higher density in YPD if supplemented with $100 \mu \mathrm{M}$ hypoxanthine. We sterilized YPD media by autoclaving. YPD overnight cultures were stored at room temperature for no more than 4-5 days prior to experiments. We used defined minimal medium SD $\left(6.7 \mathrm{~g} / \mathrm{L}\right.$ Difco $^{\mathrm{TM}}$ yeast nitrogen base w/o amino acids, $20 \mathrm{~g} / \mathrm{L}$ glucose) for all experiments [23], with supplemental metabolites added as noted. To achieve higher reproducibility than autoclaving, we sterilized SD media by filtering through $0.22 \mu \mathrm{m}$ filters.

We performed all culturing at $29.5 \pm 1^{\circ} \mathrm{C}$. lys 2 - cells were pre-grown to exponential phase in SD supplemented with excess $(164 \mu \mathrm{M})$ lysine and washed 3-5 times with SD. Where noted, we starved lys $2-$ cells for 3-6 h to deplete intracellular lysine storage. Otherwise, we did not starve lys 2 - prior to starting an experiment. ade8- cells were pre-grown to exponential phase in SD supplemented with excess hypoxanthine $(100 \mu \mathrm{M})$, washed 3-5 times 
with SD, and pre-starved in SD for $24 \mathrm{~h}$ to deplete cellular storage, unless otherwise noted.

\section{Microscope setup}

Imaging was performed using a Nikon Eclipse TE-2000U inverted fluorescence microscope. A temperature-controlled enclosure (in vivo scientific controller, model 300353) maintained the microscope at a temperature close to a set temperature $\left(29.5^{\circ} \mathrm{C}\right)$. We turned off the dark room air conditioning system since it could cause fluctuations in chamber temperature.

The microscope had a motorized stage to allow zautofocusing (Methods, "Autofocusing") and systematic xy-scanning of locations in microplate wells. Our LabVIEW (National Instruments) program controlled the microscope body, illumination, and stage position through serial port communication. The program moved the stage gently so that cells were not disturbed and individual cells could be tracked from one time point to the next. The microscope was also equipped with motorized switchable filter cubes capable of detecting a variety of fluorophores. We used an ET DsRed filter cube (Exciter: ET545/30×, Emitter: ET620/60 m, Dichroic: T570LP) for mCherry-expressing strains, and an ET GFP filter cube (Exciter: ET470/40×, Emitter: ET525/50 m, Dichroic: T495LP) for GFP-expressing strains. Fluorescence and transmitted light images were taken with a Photometrics CoolSNAP HQ [2] cooled CCD camera, interfaced with LabVIEW through the Bruxton Inc. SIDX API. Unless otherwise stated, we used a $10 \times$ objective, with a numerical aperture of 0.30 , because it provided a wide field of view while allowing easy observation of individual cells.

Image acquisition was done with an in-house LabVIEW program, incorporating autofocusing in brightfield followed by fluorescence imaging with automatically adjusted exposure time to avoid camera saturation. Optimal exposure times for fluorescence imaging may vary $(\sim 0.1-1 \mathrm{~s})$. We observed that long exposure with low-intensity light perturbed cell growth less than short exposure with high-intensity light. When we imaged ade8 - cells using a particular light configuration, the very short exposure time $(0.05 \mathrm{~s}$ for initial images) created problems for image analysis. This could be due to the latency in shutter opening/closing becoming important in short exposure times. Alternatively, since high-intensity light was used (and hence the short exposure time), exposure time had to be reduced as cells grew to avoid camera saturation, and adjusting for variable exposure time during data analysis could introduce errors. When we added a neutral density filter and/or adjusted the size of light aperture so that the exposure time was $\sim 0.3 \mathrm{~s}$, data analysis became normal. Four locations per well were imaged, with $\sim 20-200$ initial cells per image.

\section{Lid warmer}

During extended imaging, condensation could accumulate on the underside of microplate lid, even in the temperature-controlled chamber. We encountered failures in autofocusing due to heavy condensation, and condensation can degrade proper Köhlier illumination. In order to prevent condensation, we used an optically transparent heating plate to warm the lid (Figure 1B), which kept the lid temperature an average of $0.68^{\circ} \mathrm{C}$ with $2 \sigma$ of $0.22^{\circ} \mathrm{C}$ higher than the stage temperature (Supplementary Figure S1B). This eliminated condensation (Figure 1C).

Our lid warmer used an ITO glass heating plate, with an integrated thermistor temperature sensor (Oven Industries TR91-170). The $1 \mathrm{~mm}$-thick ITO glass had a $140 \mathrm{~nm}$ ITO coating (from SPI Supplies), transmitted $88 \%$ of visible light, and had a sheet resistance between $30-60 \mathrm{Ohms} / \mathrm{sq}$. We cut a $70 \mathrm{~mm} \times 120 \mathrm{~mm}$ sheet with beveled corners to match the microplate lids. To apply power for heating, we affixed strips of $5 \mathrm{~mm}$-wide conductive copper foil tape to the ITO coating along two opposite ends of the heating plate, and applied silver conductive epoxy (MG Chemicals, 8332-13G) to the tape, extending 1-2 mm onto the ITO coating to ensure a reliable connection. Wires were connected through brass conductors, which were insulated with heat shrink tubing and epoxied to the edge of the heating plate. We measured the resistance of the plate to be $20 \mathrm{Ohms}$, so the application of $5 \mathrm{~V} \mathrm{DC}$ generated $(5 \mathrm{~V})^{2} / 26 \mathrm{Ohms}=1.0 \mathrm{~W}$. This provided the needed heat when applied. The sensor readings were tested, and adjusted if necessary, using a Barnant 115 thermocouple thermometer with a $\mathrm{T}$ type probe.

The temperature of the heating plate was controlled by a LabVIEW program, with the aid of a DAQ (National Instruments USB-6008) for reading temperature sensors. Based on the measured temperatures of the heating plate and the microscope stage, the LabVIEW program activated or deactivated the heating plate when the temperature difference was $\leqslant 0.5^{\circ} \mathrm{C}$ or $\geqslant 0.8^{\circ} \mathrm{C}$, respectively. As discussed above, the heating plate was run at a fixed power of $\sim 1 \mathrm{~W}$ when active. Note: The $5 \mathrm{~V}$ supply from the DAQ was used for the sensors, and a separate supply was used for heating the plate.

\section{Sample setup}

We used flat-bottom transparent microplates with wells joined together at the bottom by a continuous sheet of plastic, such as Costar 3370 96-well plates (rather than 96-well plates where the spaces between the wells was open to the surrounding environment and thus more 
susceptible to temperature fluctuations). In these plates, air warmed by the lid warmer thermally insulates the sides of individual wells, which improves temperature uniformity.

We filled the outermost wells of a 96-well microtiter plate with water to reduce evaporation, leaving up to 60 wells for imaging. We diluted cells to low densities (1000-5000 cells inoculated in $300 \mu \mathrm{L}$ medium) to maximize our growth window and to minimize metabolite depletion during measurements. When assaying death in the absence of supplements, we added 2 10 fold more cells since there was little cell birth. We spun the plates at $2000 \mathrm{rpm}$ for $2 \mathrm{~min}$ to settle all cells to the bottom of the wells, set up the microscope as described above, and imaged the same four positions in each selected well periodically (every $0.5-2 \mathrm{~h}$ ). For each position, a brightfield and a fluorescent image was saved. For growth assays, we ran experiments until fluorescence leveled off $(16+\mathrm{h}$ for lys $2-$, and $30+\mathrm{h}$ for ade $8-$ cells $)$. For death assays, we ran experiments for up to $66 \mathrm{~h}$. We found that growth rates for the same samples did not vary significantly across different well positions in a plate (Supplementary Figure S2).

\section{Autofocusing}

Automated imaging of the cells was performed using custom software written in LabVIEW. At each time point, it was necessary to auto-focus on the cells (in bright-field) due to the small vertical drift caused by small changes in temperature or mechanical stress. At the beginning of an experiment, manual focusing was performed on four corner sample wells to ensure that the plate was level (otherwise, we needed to adjust the screw positions of the plate holder). Then, coarse auto-focusing was performed for one position in each sample well at \pm 100 z-positions spaced at $3 \mu \mathrm{m}$ apart, and the best focal plane was chosen to initiate an experiment. To identify the best focal plane for imaging and to prevent a loss of focus during the experiment, fine auto-focusing ( $\pm 30 \mathrm{z}$-positions spaced at $2 \mu \mathrm{m}$ apart) was performed for each image location at each time point.

Each 16-bit, grayscale image was imported directly into LabVIEW, and converted into a two-dimensional array of real numbers. The optimal z-position for focusing was chosen using a variant of the Brenner auto-focus algorithm [24,25]. The quality of focus, $A(z)$, was measured by computing the average of the squared horizontal and vertical gradient:

$$
\begin{gathered}
A(z)=\sum_{i=1}^{N-2}(p(i+2, j)-p(i, j))^{2} \\
+\sum_{j=1}^{M-2}(p(i, j+2)-p(i, j))^{2},
\end{gathered}
$$

where $N \times M$ were the dimensions of each image, and $p(i, j)$ was the pixel intensity at row $i$ and column $j$. Local maxima in $A(z)$ corresponded to sharply-focused images (in bright-field). Comparison between data two pixels apart rather than adjacent pixels reduced the effects of correlated noise and the natural illumination of nearby pixels.

When observing yeast we often observed multiple local maxima in the range of $z$ positions (Supplementary Figure S12A). Assuming yeast cells behave like small spherical lenses of diameter $D \approx 5 \mu \mathrm{m}$, then this phenomenon is likely a complex function of reflection, refraction, and diffraction [24]. However, some qualitative features may be illustrated by simply considering refraction of a ball lens, where the effective focal length $(E F L)$ is Ref. [22]:

$$
E F L=\frac{n_{c} D}{4\left(n_{c}-n_{w}\right)},
$$

where $n_{w}=1.33$ is the index of refraction of the surrounding water, and $n_{c}$ is the index of refraction of the cell. Supplementary Figure S12 shows that local maximum (iii) was due to light that was focused approximately $14 \mu \mathrm{m}$ from the yeast cells, which would correspond to $n_{c}=1.46$. This is consistent with previous measurements showing that $n_{c} \approx 1.53$ [27], although we note that this value depends on the size and density of the cells. The second maximum (i) was located at an image plane below the cells that contained light halos from the apparent source of the focused light.

We found that the local minimum in $A(z)$ between these two maxima (ii) conveniently corresponded to a focal plane adequate for identifying and imaging fluorescent cells (Supplementary Figure S12). Thus, we chose this local minimum for imaging in all of our experiments. The three points closest to the minimum were fit to a parabola, and the minimum of the parabola was chosen as the optimal focus position. The microscope stage was then moved to the optimal focal plane for bright-field and fluorescence imaging. A similar method for autofocusing, i.e., using the local minimum in the autofocus score, has been used before [28].

\section{Individual cell tracking at low metabolite concentrations}

At very low concentrations of supplements and low cell densities, birth and death events could be counted. In manual counting, we scanned through fluorescent images in ImageJ (e.g., Supplementary Movies 2-3). After counting the initial number of cells in an image, we proceeded through each image and noted the appearance or disappearance of cells. We counted a birth event as the appearance of a new cell adjacent to a cell present in the 
previous image. We differentiated this from two vertically-stacked cells shifting orientation to create the appearance of a new cell birth, as the fluorescence intensity of stacked cells dropped noticeably during orientation shift. We counted a death event when a cell present in the previous image suddenly lost fluorescence. Occasionally cellular fluorescence slowly faded over time rather than disappearing suddenly, and in this case we counted the initial drop in intensity as the death of the cell.

We also automated live and dead cell counting to analyze time-lapse bright-field and fluorescent images. The ImageJ plugin CellQuant was written by Adam Waite and is available at https://github.com/nodice73/ImageJ/ blob/master/CellQuant.txt. For each cell sample, we took an off-focus bright-field image to create a dark ring around each cell (Supplementary Figure S12, case iii; Supplementary Figure S13A) and an in-focus fluorescence image. The plugin then created a mask in the brightfield image to separate the background from the cellcontaining foreground. Specifically, low frequency intensity information (e.g., due to non-uniform illumination) was removed using the "rolling ball" background subtraction [29,30]. The bit depth of the image was reduced to 8 (since it gave more consistent thresholding values from image to image) and the image was blurred with a Gaussian filter with a standard deviation of 1 to reduce high frequency noise caused by, for example, shot noise in camera. A variance filter with a radius of 3 was used to identify edges. The image was duplicated and thresholded using both "RenyiEntropy" and "MaxEntropy" algorithms [31], since one of the two algorithms would work well depending on cell density. To create a mask, all pixel intensities greater than the threshold value were assigned 1 , and the rest assigned 0 . Sometimes, spurious noise would cause a small group of white pixels (much smaller than cell size) in the middle of black background, and such small holes were filled. Then, two selections were created. Since a selection can represent either background or cell-containing foreground, the standard deviation of each selection was calculated, and the selection with the lower standard deviation was used as the background selection. Among the two thresholds created by "RenyiEntropy" and "MaxEntropy", the plugin selects the threshold defining the larger background area (which is less prone to artifacts). Once the background selection was determined, the average background value of the original bright-field image was calculated.

To select individual cells, the average background value calculated above was used to alter the pixel intensity of the original bright-field image (details in Supplementary Figure S13B). A Gaussian blur with standard deviation of 0.5 was then applied to the modified bright-field image (Supplementary Figure S13B) to reduce high frequency noise. To select individual cells, the "Find Maxima" algorithm was run on the altered and blurred bright-field image, with output set to "Segmented Particles". The "Analyze Particles..." algorithm was then run on the segmented particles, and the selections added to the ROI manager. The cell selections (including the dark ring) were applied to the original bright-field image, and those with low variance were rejected as background (Supplementary Figure S13C).

After individual cells were selected, each cell's fluorescence was quantified in the fluorescence image. To do this, low frequency intensity information was removed in fluorescence image using the rolling ball background subtraction, and spurious bright pixels were removed with the "Remove Outliers..." command. A background mask in the fluorescence image was made as described for bright-field, and the selection made from this mask was used to define an average background for the fluorescence image. The average background plus a user-defined number of standard deviation of this background (1 standard deviation was used here) were subtracted from the fluorescence image. Values below zero were set to zero. Cell selections from bright-field image were then applied to this background-subtracted fluorescence image (Supplementary Figure S13D). Cell selections from bright-field containing zero fluorescence were counted as dead cells, and all other cell selections were considered live.

These counts yielded similar results to the manual counting procedure described above and could be applied to make the assay higher-throughput. CellQuant consistently under-counts cells by $\sim 10 \%$ as compared to manual counting (Supplementary Figure S5), presumably due to differences in the definition of a cell - a budded cell is counted as two cells in manual counting and as one cell by CellQuant. All counting data presented in this paper are from manual counting unless otherwise noted.

\section{Fluorescent image analysis}

We analyzed time-lapse images using Bioact, an ImageJ [32] plugin written by Adam Waite (Supplementary Figure S14, available at https://github.com/nodice73/ Java/tree/master/imagej_plugins/bioact). Bioact measured the background-subtracted total fluorescence intensity of all cells in an image. To distinguish fluorescent cells from background, each fluorescent image was blurred with a Gaussian filter using a standard deviation $(\sigma)$ of 1 pixel. Low frequency noise was removed using the "Rolling Ball" background subtraction algorithm $[29,30]$. The dynamic range of the fluorescent image was reduced from 16-bit to 8-bit, and each pixel was replaced by the maximum value in a 3 pixel radius. The resulting image was thresholded using the "MaxEntropy" method 
[31] for low density images or the "IsoData" method [33] for higher-density images and converted to a binary mask [32]. After filling holes using binary closing, the mask was dilated by 2 pixels. From this mask, the percent pixels considered foreground was calculated. Each mask was then applied to the un-manipulated original image, and the foreground and background intensities were measured. At low cell densities, the background was calculated for each image and subtracted. We found that when the foreground made up more than a specified fraction of the total image area, the background estimate was no longer accurate, and the running average background value calculated before this threshold was met was used as the background value for subsequent images. This method occasionally failed if cells were nearly confluent during late stage growth. However, we were only interested in maximum growth rate, which occurred before confluency.

We plotted background-subtracted fluorescence intensity over time for all four positions in each well to allow visual inspection. In rare occasions, all four positions were out-of-focus and none were used. In a small subset of experiments, a discontinuous jump in data appeared in all four positions for reasons we do not understand. We did not calculate rates across the jump. Occasionally, data from one or two positions deviated from the rest. This could have been due to a number of reasons, including spurious shifts in stage position, or black or bright dust particles in the field of view. In these cases, we inspected the images, and outliers with obvious causes were excluded. If the fluorescence dynamics of four positions differed due to cell heterogeneity at low concentrations of metabolites, all positions were retained.

We normalized intensity against that of time zero, and averaged across positions. We calculated growth rate over three to four consecutive time points, and plotted the maximal growth rate against metabolite concentration. If maximal growth rate occurred at the end of an experiment, then the experimental duration was too short and data were not used.

\section{Bioassay}

We used a bioassay to quantify lysine concentrations from supernatants. In order to obtain supernatant, we filtered cell cultures through a $0.45 \mu \mathrm{m}$ nitrocellulose filter and stored supernatant at $-80^{\circ} \mathrm{C}$ until quantification. We mixed $150 \mu \mathrm{L}$ supernatant sample with an equal volume of master mix containing $2 \times \mathrm{SD}$ and lysine-requiring tester cells $\left(\sim 1 \times 10^{4}\right.$ cells $/ \mathrm{mL}$ WY2270 $)$ in a flat-bottom 96-well plate. We measured growth rates of WY2270 in unknown samples and compared them to growth rates of WY2270 run concurrently with known concentrations of lysine. The growth rate scaled linearly with lysine concentration up to $1 \mu \mathrm{M}$ (i.e., $2 \mu \mathrm{M}$ in undiluted sample, Supplementary Figure S15). From this standard curve, we inferred lysine concentrations of samples. Assay sensitivity was $0.1 \mu \mathrm{M}$.

\section{Flow cytometry}

We first prepared bead standards for quantifying cell density. Fluorescent beads (ThermoFisher Cat R0300, $3 \mu \mathrm{m}$ red fluorescent beads) were autoclaved in a factoryclean glass tube, diluted into sterile $0.9 \% \mathrm{NaCl}$, and supplemented with sterile-filtered Triton X-100 to a final $0.05 \%$ (to prevent beads from clumping). We sonicated beads and kept them in constant rotation to prevent settling. We quantified bead concentrations by counting beads using a hemacytometer and a BD Coulter counter. The final bead stock was generally $4 \sim 8 \times 10^{6} / \mathrm{mL}$.

Culture samples were diluted to OD $0.01-0.1\left(7 \times 10^{5}-\right.$ $7 \times 10^{6} / \mathrm{mL}$ ) in MilliQ $\mathrm{H}_{2} \mathrm{O}$ in unautoclaved $1.6 \mathrm{~mL}$ Eppendorf tubes. In a 96-well plate, $90 \mu \mathrm{L}$ sample was supplemented with $10 \mu \mathrm{L}$ bead stock to calculate cell density from cell:bead ratio. We also added $2 \mu \mathrm{L}$ of $1 \mu \mathrm{M}$ nucleic acid dye ToPro3 (Molecular Probes T-3605) which stains dying/dead cells with compromised membrane. Flow cytometry was performed on Cytek DxP Cytometer equipped with four lasers, ten detectors, and an autosampler. Fluorescent tags GFP, mCherry, and ToPro were respectively detected by $50 \mathrm{~mW} 488 \mathrm{~nm}$ laser with 505/10 (i.e., 500-515 nm) detector, $75 \mathrm{~mW} 561 \mathrm{~nm}$ Laser with $615 / 25$ detector, and $25 \mathrm{~mW} 637 \mathrm{~nm}$ laser with 660 / 20 detector. Each sample was run in triplicate and individually analyzed using FlowJo ${ }^{\circledR}$ software to identify numbers of events of beads, dead cells, and various live fluorescent cells. We calculated the mean cell density from triplicate measurements, with the coefficient of variation generally within 5\%-10\%.

\section{Chemostat culturing}

We constructed an eight-vessel chemostat with a design [15] modified from an existing multiplexed culturing device [34]. A chemostat creates a nutrient-limited environment where the population is forced to grow at a constant, pre-determined rate slower than the maximal growth rate [35].

Specifically, a medium containing a limiting metabolite is added to the culturing chamber at a constant flow rate $f$ $(\mathrm{mL} / \mathrm{h})$. The culture effluent is removed from the chamber at the same rate $f$, thereby maintaining a constant culture volume $V$. Mathematically [35], live population density $N$ satisfies

$$
\mathrm{d} N / \mathrm{d} t=(r-f / V) N,
$$

where $r\left(\mathrm{~h}^{-1}\right)$ is the growth rate (birth rate minus death 
rate), $f$ is the flow rate, and $f / V$ is the dilution rate $\left(\mathrm{h}^{-1}\right)$. At steady state, the growth rate and the dilution rate are equal:

$$
r=f / V \text {. }
$$

The limiting metabolite also reaches a steady state in the culturing vessel. Thus, the steady state concentration of the limiting metabolite supports a growth rate equaling the dilution rate.

Due to rapid evolution, we designed experiments so that live and dead populations quickly reached steady state and the experiment lasted $\leqslant 26 \mathrm{~h}$ [13]. Specifically, we washed exponentially-growing cells to remove extracellular lysine and inoculated $1 / 4-1 / 2$ of the volume at $1 / 3$ of the expected steady-state density. We filled the rest of the $19 \mathrm{~mL}$ chamber with reservoir media (resulting in less than the full $20 \mu \mathrm{M}$ of reservoir lysine, but more than enough for maximal initial growth rate, $\sim 10-15 \mu \mathrm{M}$ ). We sampled cultures periodically to track population dynamics using flow cytometry (Supplementary Figure S9A) and filtered supernatant through a $0.45 \mu \mathrm{m}$ nitrocellulose filter. We froze the supernatants for metabolite quantification at the conclusion of an experiment (Supplementary Figure S9B).

\section{SUPPLEMENTARY MATERIALS}

The supplementary materials can be found online with this article at https:// doi.org/10.1007/s40484-018-0160-7.

\section{ACKNOWLEDGEMENTS}

We thank Jose Pineda for performing the experiment in Supplementary Figure $\mathrm{S} 3$ and Li Xie for consultation regarding models.

\section{COMPLIANCE WITH ETHICS GUIDELINES}

The authors Samuel F. M. Hart, David Skelding, Adam J. Waite, Justin C. Burton and Wenying Shou declare that they have no conflict of interests.

All procedures performed in studies involving animals were in accordance with the ethical standards of the institution or practice at which the studies were conducted, and with the 1964 Helsinki declaration and its later amendments or comparable ethical standards.

\section{REFERENCES}

1. Müsken, M., Di Fiore, S., Römling, U. and Häussler, S. (2010) A 96-well-plate-based optical method for the quantitative and qualitative evaluation of Pseudomonas aeruginosa biofilm formation and its application to susceptibility testing. Nat. Protoc., 5, 1460-1469

2. Ziv, N., Siegal, M. L. and Gresham, D. (2013) Genetic and nongenetic determinants of cell growth variation assessed by highthroughput microscopy. Mol. Biol. Evol., 30, 2568-2578

3. Shi, H., Colavin, A., Lee, T. K. and Huang, K. C. (2017) Strain Library Imaging Protocol for high-throughput, automated single- cell microscopy of large bacterial collections arrayed on multiwell plates. Nat. Protoc., 12, 429-438

4. Boutros, M., Heigwer, F. and Laufer, C. (2015) Microscopy-based high-content screening. Cell, 163, 1314-1325

5. Monod, J. (1949) The growth of bacterial cultures. Annu. Rev. Microbiol., 3, 371-394

6. Kargi, F. and Shuler, M. L. (1979) Generalized differential specific rate equation for microbial growth. Biotechnol. Bioeng., 21, 18711875

7. Kovárová-Kovar, K. and Egli, T. (1998) Growth kinetics of suspended microbial cells: from single-substrate-controlled growth to mixed-substrate kinetics. Microbiol. Mol. Biol. Rev., 62, 646666

8. Shou, W., Ram, S. and Vilar, J. M. (2007) Synthetic cooperation in engineered yeast populations. Proc. Natl. Acad. Sci. USA, 104, 1877-1882

9. Momeni, B., Chen, C.-C., Hillesland, K. L., Waite, A. and Shou, W. (2011) Using artificial systems to explore the ecology and evolution of symbioses. Cell. Mol. Life Sci., 68, 1353-1368

10. Waite, A. J. and Shou, W. (2012) Adaptation to a new environment allows cooperators to purge cheaters stochastically. Proc. Natl. Acad. Sci. USA, 109, 19079-19086

11. Momeni, B., Brileya, K. A., Fields, M. W. and Shou, W. (2013) Strong inter-population cooperation leads to partner intermixing in microbial communities. eLife, 2, e00230

12. Momeni, B., Waite, A. J. and Shou, W. (2013) Spatial selforganization favors heterotypic cooperation over cheating. eLife, 2, $\mathrm{e} 00960$

13. Hart, S. F. M., Mi, H., Green, R., Pineda, J. M. B., Momeni, B., Shou, W. (2018) Resolving challenges in quantitative modeling of microbial community dynamics. bioRxiv 356519

14. Wiemken, A. and Dürr, M. (1974) Characterization of amino acid pools in the vacuolar compartment of Saccharomyces cerevisiae. Arch. Microbiol., 101, 45-57

15. Skelding, D. H., Hart, S. F. M., Vidyasagar, T., Pozhitkov, A. E. and Shou, W. (2018) Developing a low-cost milliliter-scale chemostat array for precise control of cellular growth. Quant. Biol., 6, 129-141

16. Boer, V. M., Amini, S. and Botstein, D. (2008) Influence of genotype and nutrition on survival and metabolism of starving yeast. Proc. Natl. Acad. Sci. USA, 105, 6930-6935

17. Puig, S., Lee, J., Lau, M. and Thiele, D. J. (2002) Biochemical and genetic analyses of yeast and human high affinity copper transporters suggest a conserved mechanism for copper uptake. J. Biol. Chem., 277, 26021-26030

18. Xiao, C. and Cant, J. P. (2003) Glucose transporter in bovine mammary epithelial cells is an asymmetric carrier that exhibits cooperativity and trans-stimulation. Am. J. Physiol. Cell Physiol., 285, C1226-C1234

19. Casillas, T., Delicado, E. G., García-Carmona, F. and MirasPortugal, M. T. (1993) Kinetic and allosteric cooperativity in Ladenosine transport in chromaffin cells. A mnemonical transporter. Biochemistry, 32, 14203-14209

20. Lin, C. H., MacGurn, J. A., Chu, T., Stefan, C. J. and Emr, S. D. 
(2008) Arrestin-related ubiquitin-ligase adaptors regulate endocytosis and protein turnover at the cell surface. Cell, 135, 714-725

21. Jones, C. B., Ott, E. M., Keener, J. M., Curtiss, M., Sandrin, V. and Babst, M. (2012) Regulation of membrane protein degradation by starvation-response pathways. Traffic, 13, 468-482

22. Waite, A. J. and Shou, W. (2014) Constructing synthetic microbial communities to explore the ecology and evolution of symbiosis. Methods Mol. Biol., 1151, 27-38

23. Guthrie, C. and Fink, G. R. (1991) Guide to Yeast Genetics and Molecular Biology. 1st, Ed., Academic Press

24. Mir, H., Xu, P. and Van Beek, P. (2014) An extensive empirical evaluation of focus measures for digital photography. In Proceedings of SPIE -The International Society for Optical Engineering. 9023

25. Brenner, J. F., Dew, B. S., Horton, J. B., King, T., Neurath, P. W. and Selles, W. D. (1976) An automated microscope for cytologic research a preliminary evaluation. J. Histochem. Cytochem., 24, 100-111

26. Smith, A. E., Zhang, Z. and Thomas, C. (2000) Wall material properties of yeast cells: part 1. Cell measurements and compression experiments. Chem. Eng. Sci., 55, 2031-2041

27. Kim, M.-S., Scharf, T., Mühlig, S., Fruhnert, M., Rockstuhl, C., Bitterli, R., Noell, W., Voelkel, R. and Herzig, H. P. (2016) Refraction limit of miniaturized optical systems: a ball-lens example. Opt. Express, 24, 6996-7005

28. Gordon, A., Colman-Lerner, A., Chin, T. E., Benjamin, K. R., Yu, R. C. and Brent, R. (2007) Single-cell quantification of molecules and rates using open-source microscope-based cytometry. Nat. Methods, 4, 175-181

29. Hartig, S. M. (2013) Basic image analysis and manipulation in ImageJ. Curr. Protoc. Mol. Biol. 102, 14.15.1-14.15.12

30. Sternberg, S. R. (1983) Biomedical image processing. Computer, $16,22-34$

31. Kapur, J. N., Sahoo, P. K. and Wong, A. K. (1985) A new method for gray-level picture thresholding using the entropy of the histogram. Comput. Vis. Graph. Image Process., 29, 273-285

32. Schneider, C. A., Rasband, W. S. and Eliceiri, K. W. (2012) NIH Image to ImageJ: 25 years of image analysis. Nat. Methods, 9, 671-675

33. Ridler, T. and Calvard, S. (1978) Picture thresholding using an iterative selection method. IEEE Trans. Syst. Man Cybern., 8, 630632

34. Takahashi, C. N., Miller, A. W., Ekness, F., Dunham, M. J. and Klavins, E. (2015) A low cost, customizable turbidostat for use in synthetic circuit characterization. ACS Synth. Biol., 4, 32-38

35. Novick, A. and Szilard, L. (1950) Experiments with the Chemostat on spontaneous mutations of bacteria. Proc. Natl. Acad. Sci. USA, 36, 708-719 